\title{
Análisis de falla del árbol de levas de un motor de seis cilindros en línea
}

\author{
Gabriel E. Téllez Fontecha, \\ Ing. Metalúrgico y de Materiales, \\ Universidad Industrial de Santander (UIS), \\ Consultor Independiente, Colombia. \\ gabotellez.uis@gmail.com
}

\author{
Jorge Guillermo Díaz R., \\ Máster of Science in Engineering Technology, \\ University of North Texas (UNT), Grupo AMSCP, \\ Universidad Santo Tomás Bucaramanga, Colombia: \\ djgdiaz@ustabuca.edu.co
}

\begin{abstract}
Resumen - En este trabajo se analiza la posible causa de la falla de un árbol de levas de un motor de combustión interna proveniente de motor de seis cilindros en línea. Un análisis morfológico muestra micro grietas, provenientes de un conducto central, marcas de maquinado severo y defectos probablemente producidos por maquinado de desbaste rápido y severo en zonas críticas de diseño y altos concentradores de esfuerzo. Sumado a esto se presentan, y debido a la naturaleza de las cargas de trabajo de un eje de levas, en los puntos de concentración de esfuerzos, esfuerzos fluctuantes que según el análisis realizado se deduce que no indujeron una falla por fatiga. Se realizó un análisis de Fluorescencia de Rayos X (XRF) para establecer el material del cual estaba fabricado el eje. Este análisis comprueba las recomendaciones para selección de materiales dadas por varios autores en el área de diseño de ejes. Con base en los datos técnicos del vehículo, se establecieron las cargas a las que estaba sometido el árbol de levas. Por último se utiliza el criterio de Griffith para estimar un punto de falla. La presencia simultánea de estos factores disminuyó la resistencia efectiva del eje, creó las condiciones adecuadas para la fractura en forma frágil y abrupta.
\end{abstract}

Palabras clave - árbol de levas, falla frágil, esfuerzos fluctuantes, análisis de falla.

\footnotetext{
Abstract - This paper discusses a possible cause for an internal combustion engine camshaft failure in a six-cilinder internal combustion engine. A morphological analisis showed that there were micro cracks from a central conduit, defects probably caused by a severe machinning process, and machining defects in design critical areas with a high stress concentration factor. Additionally, and due to the nature of solicitations present in a camshaft, there were stresses present due to the fluctuating nature of the axis work at concentration points. XRF was done to establish the compostion and therefore the camshaft's steel mechanical properties. Such analisis coincides with several authors' recommendation for materials selection for this kind of shafts. The vehicle's technical seet provided the maximum torque that was ued to determine stresses present in the shaft. The Griffith criterion was used to establish a falilure value. The combined presence of the aforementioned factors reduced the shaft effective resistance creating conditions for a brittle and abrupt fracture.
}

Keywords - Camshaft, brittle failure, fluctuating stress, failure analisys

\section{INTRODUCCIÓN}

En un motor de 4 ciclos, cada uno de los cilindros es provisto con una o dos válvulas de admisión y válvulas de escape. El eje de levas permite abrir y cerrar las válvulas [9]. La cima en la leva empuja para abrir la válvula y la zona baja permite que la válvula esté cerrada por la fuerza de un resorte [10]. Los ejes de levas están fabricados generalmente en aceros de medio contenido de carbono, con el fin de realizarles temple superficial por inducción $u$ otros métodos similares en las superficies de las levas y obtener altos valores de dureza que asegure la resistencia al desgaste pero que no sean muy duros al maquinar. De otro lado, en los muñones y el núcleo del eje es deseable una estructura perlítica que garantice tenacidad [4]. De esto podemos deducir que las cargas en un árbol de levas generan esfuerzos de flexión, torsión y cortante, por lo que se generan estados triaxiales de esfuerzos. Debido a la rotación a la que está sometido el eje, esfuerzos por fatiga están presentes [11]. Al analizar las cargas sobre el eje vemos cómo la potencia se transmite por medio de las levas, donde las cargas ejercidas sobre ellas son equivalentes a un sistema fuerza - par aplicados en el centro de las correspondientes secciones transversales del eje [7]. Esto implica que el eje está sometido tanto a carga transversal como a carga torsional. Sin embargo, para efectos de cálculo, los esfuerzos cortantes producidos en el eje por las cargas transversales, que como son más pequeños que los producidos por torques, generalmente son despreciados $[1,6]$. Por facilidad de diseño y manufactura el eje se fabrica integralmente con las levas maquinadas sobre el eje. En 
la Figura 1 se aprecia una sección típica de un árbol de levas.

FIG. 1. EJE DE LEVAS CON LEVAS MAQUINADAS SOBRE EL ÁRBOL. EL PERFIL DE LA LEVA DETERMINA EL MOVIMIENTO DE APERTURA DE LA VÁLVULA Y EL TIEMPO DE APERTURA.

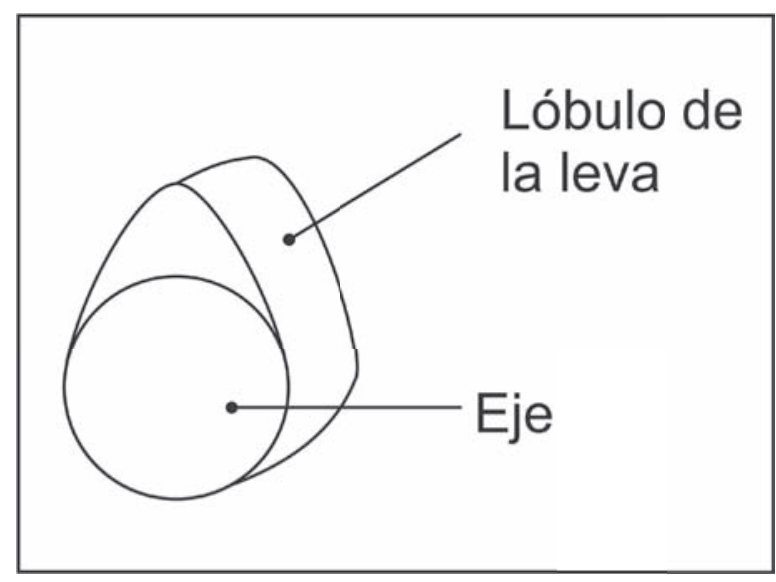

Fuente: Los autores

\section{MATERIALES Y MÉTODOS}

\section{A. Metodología}

El análisis de la falla ocurrida al árbol de levas empezó al realizar una observación macroscópica de las partes y las superficies de fractura. Inicialmente fueron identificados los muñones de bancada (a, b, c, d, e, f) y las levas (1, 2, 3, 4, 5, 6, 7, 8, 9, $10,11,12)$, los cuales fueron numerados como se muestra en la Figura 2.

FIG.2. IDENTIFICACIÓN Y NOMENCLATURA DE LA SECCIÓN FALLADA

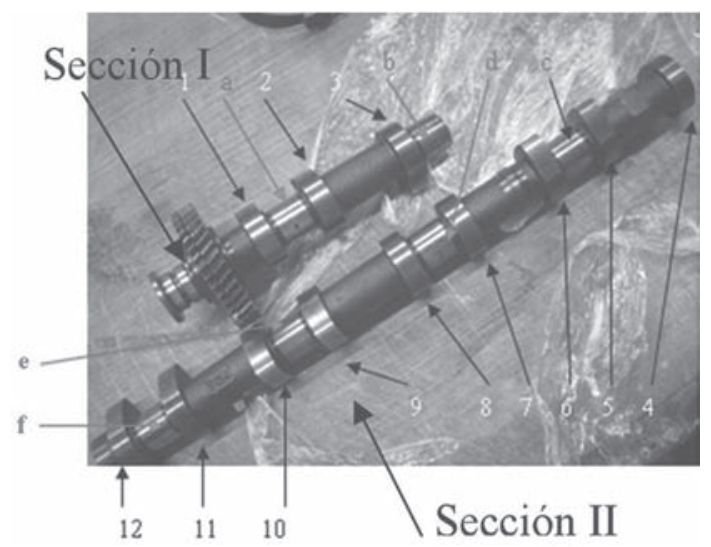

Fuente: Los autores

En la Figura 2 se puede apreciar la posición de la falla respecto a la longitud total del árbol de levas. De acuerdo con la numeración propuesta, la falla ocurrió en el muñón de bancada b. La falla se pre- sentó cuando se completaron aproximadamente $20,000 \mathrm{Km}$. (2 años de servicio). Igualmente se aprecia la nomenclatura de las secciones, I para la sección más corta y que contiene el muñón y más cercana al engranaje de transmisión. Sección II para la más larga y que empieza justo en el borde de la leva donde ocurrió la falla.

Las fotografías 1 al 8 (excepto No. 2) fueron tomadas con una cámara comercial SONY Cibershot 8.1 Megapixels. Las fotografías 7 a 13 fueron tomadas con un Microscopio Cofocal 3D HIROX, del laboratorio de Bio-materiales de la Universidad Industrial de Santander. La composición química fue evaluada con Fluorescencia de Rayos $X$ (XRF) realizada en el CDP (Centro de Desarrollo Productivo) de joyería en Bucaramanga.

La observación de las superficies de los muñones y las levas del árbol de levas permitió valorar la calidad de los acabados superficiales de mecanizado y se observó que las superficies no presentaban desgaste excesivo ni ninguna otra evidencia física de deterioro. Además el ensamble fue adecuado y no existían reparaciones previas. No se evidenció picado o desprendimiento de material. Para la ubicación de defectos se numeró la sección como se muestra en la Figura 3.

Por la geometría del pedazo de cigüeñal desprendido en la fractura con el muñón 1 se aprecia que la falla ocurrió cercana al lado del engranaje. En este extremo se manifiesta la entrega del torque hacia el resto del eje. La región de la falla está localizada en la parte final del muñón de apoyo de bancada "b" del eje próximo a la leva " 4 ". FIG 3. NOMENCLATURA PARA LA UBICACIÓN DE DEFECTOS EN LA
SECCIÓN FALLADA DEL LADO DE LA LEVA

\section{$\begin{array}{llll}A & \text { B } & \text { C } & \text { D }\end{array}$}

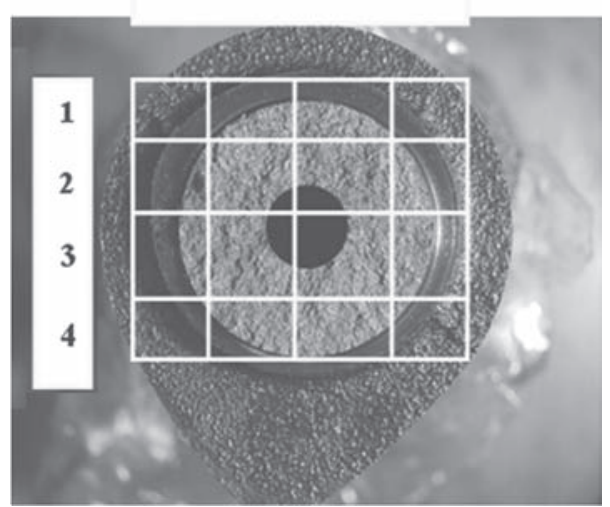

Fuente: Los autores 
La Figura 4 muestra el eje de levas por el lado de la bancada b, sección I.

FIG.4. EJE DE LEVAS POR EL LADO DE LA BANCADA “B”

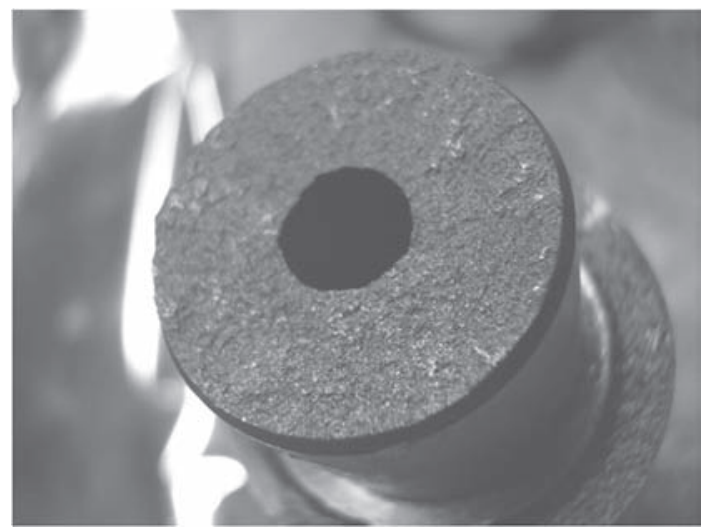

Fuente: Los autores

\section{B. Descripción}

En la Figura 3 sección I, se aprecian dos zonas de desprendimientos de material en la superficie lateral del muñón de bancada. Además se aprecia también un ligero desgaste sobre la sección circular en la cual se encuentra ubicado el orifico de lubricación. Esto posiblemente atribuible a impurezas sólidas, polvo, suciedad, partículas abrasivas o partículas metálicas presentes en el aceite y que posiblemente entraron por el filtro de aire. Dichas partículas se incrustaron en la superficie del cojinete, rozan el eje y crean puntos de fricción localizados y provocan ruptura de película de aceite con el correspondiente desprendimiento de material. Esta situación se puede apreciar más claramente en las Figuras 5 y 6 .

FIG.5. EJE DE LEVAS VISTO POR EL LADO DE LA BANCADA “B”, SECCIÓN I UBICADA LATERALMENTE EN C1 EN DONDE SE APRECIAN GRIETAS

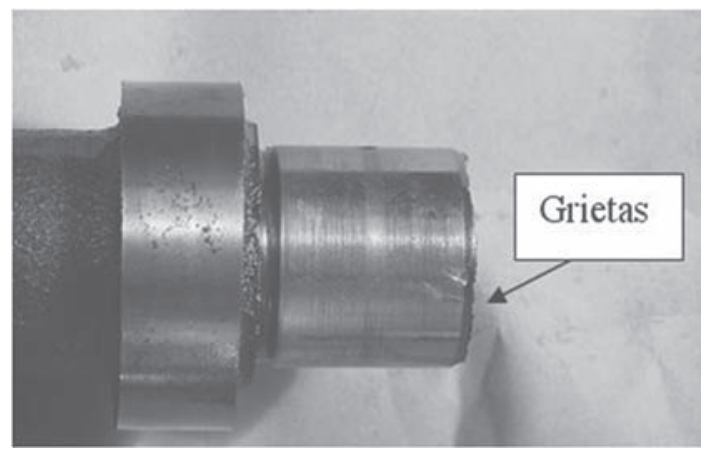

Fuente: Los autores
FIG.6. EJE DE LEVAS VISTO POR EL LADO DE LA BANCADA “B”, SECCIÓN I UBICADA LATERALMENTE EN B1 EN DONDE SE APRECIAN GRIETAS

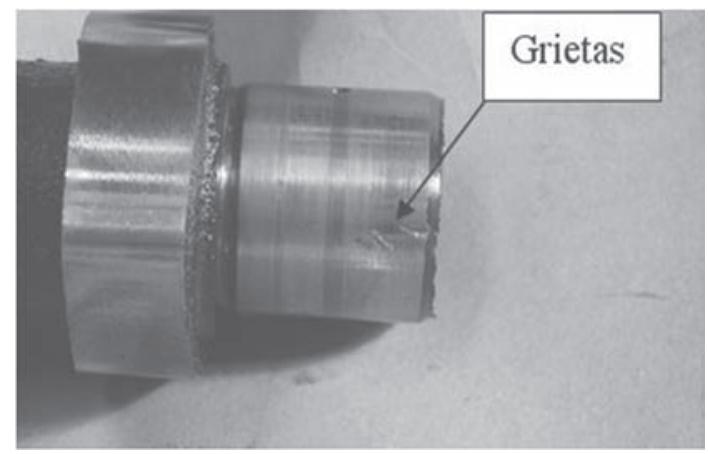

Fuente: Los autores

En la observación detallada de las superficies de fractura como la que se muestra en la Figura 7 , se reconocieron diversas grietas las cuales emanaban del conducto central del eje. No hay presencia de características morfológicas típicas de las fallas por esfuerzos de fatiga, como son las denominadas marcas de playa ni superficies lisas. La Figura 10 muestra la superficie de falla donde se observan puntos de posibles inicios de falla cercanos al concentrador de esfuerzos 1 (conducto central del eje), la cual avanzó hacia el exterior del eje.

FIG.7.SECCIÓN II MUESTRA MICRO GRIETAS QUE PARTEN DESDE EL CONDUCTO CENTRAL DEL ÁRBOL

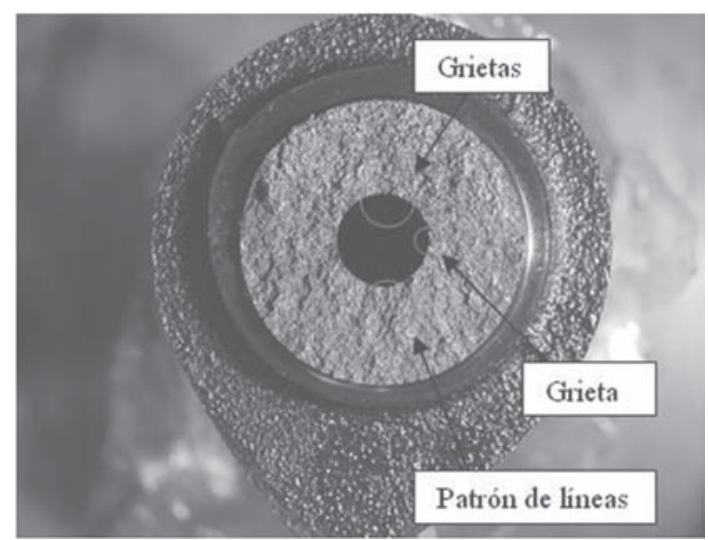

Fuente: Los autores

Al observar bajo mayores aumentos (20x) se encontró la presencia de grietas sobre las dos superficies. En la Figura 8 se observa la propagación de una grieta transversal proveniente de la anomalía ubicada lateralmente en C1 ya referenciada en la Figura 5. 
FIG.8. GRIETA TRANSVERSAL PROVENIENTE DE C1.

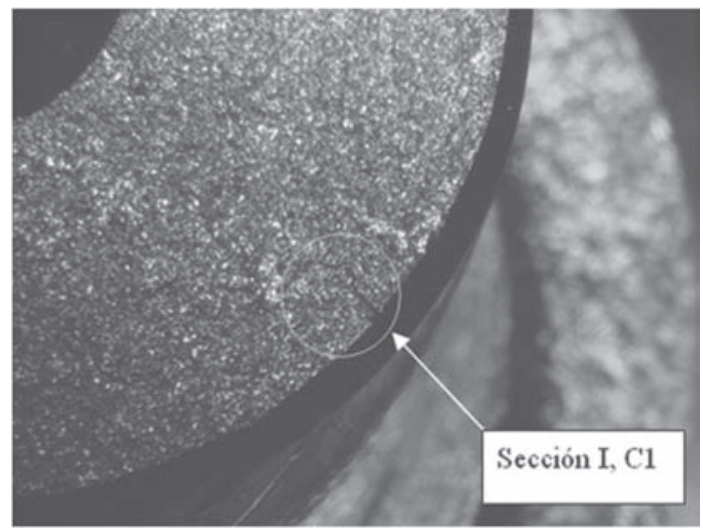

Fuente: Los autores

En la Figura 9 se notan claramente los puntos iniciales de la falla que parten radialmente del conducto central hacia el exterior del eje, lo cual sugiere una falla por torque excesivo o sobrecarga, el cual debido a la naturaleza del material, falla frágilmente. La no presencia de patrones de propagación de las grietas indica una falla abrupta y frágil, lo cual indica que la sobrecarga no permitió la propagación de dichas grietas.

FIG. 9. SECCIÓN I B3, $20 X$

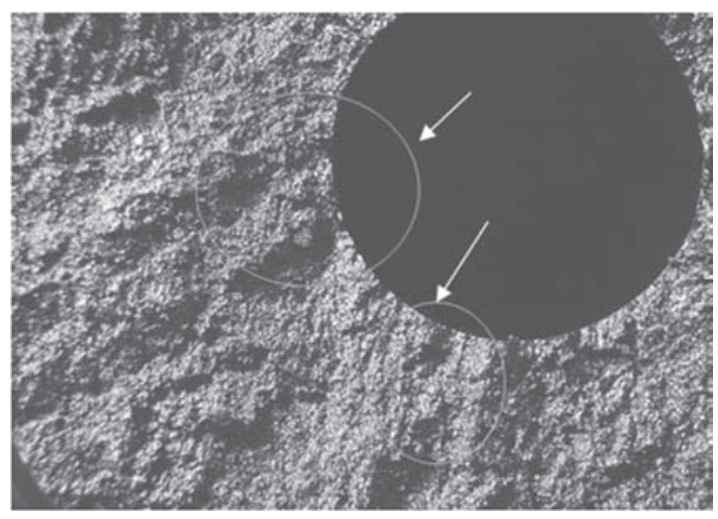

Fuente: Los autores

En la Figura 11 se aprecia una gran cantidad de entallas provocadas muy posiblemente por un maquinado severo. La presencia de estos concentradores de esfuerzos afectan aún más la capacidad de resistir carga del material.
FIG.11. B1 SECCIÓN 1. MARCAS DE MAQUINADO DURANTE LA FABRICACIÓN, 20X

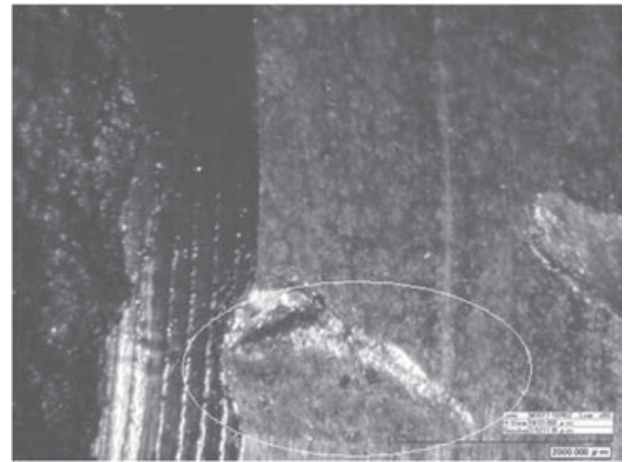

Fuente: Los autores

Consecuentemente con esto en la Sección II se observa un patrón similar al de la sección I. Las grietas se hacen más notorias y vemos claramente patrones de propagación radiales hacia el exterior del eje como se muestra en la Figura 11.

FIG.11. SECCIÓN II, 20X

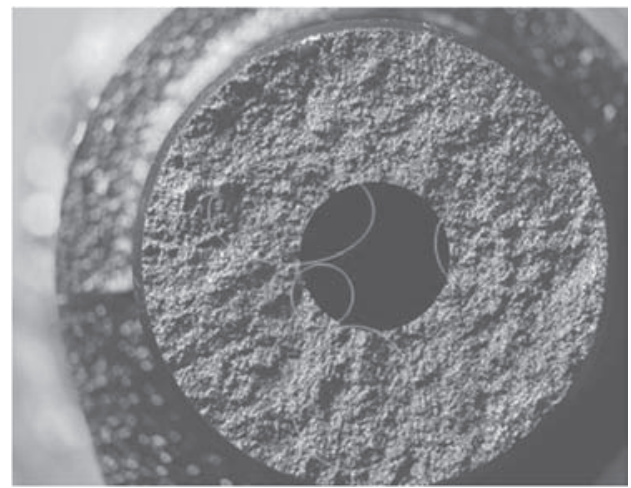

Fuente: Los autores

En la Sección II también se encuentran entallas debido al maquinado de desbaste, como se aprecia en la Fig. 12.

FIG.12 ENTALLAS DEBIDO A MAQUINADO SECCIÓN II, 20X

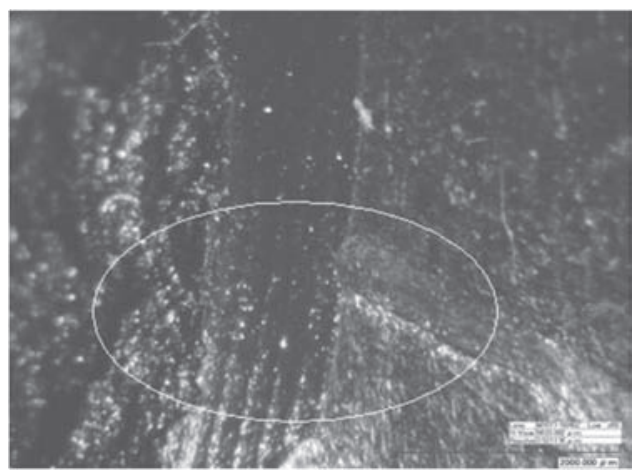

Fuente: Los autores 


\section{DISCUSIÓN}

\section{A. Modelo Teórico de Esfuerzos}

Debido a que el eje está sometido a torque y cargas cíclicas, se presenta un mecanismo de deslizamiento atómico localizado [12]. En este caso la fractura comenzó alrededor del conducto central, zona de alta concentración de esfuerzos. Debido a esto la resistencia al cortante del material del eje fue superada por los esfuerzos cortantes. Meyers, M. y Chawla, K. [11] afirman que en presencia de esfuerzos cíclicos a medida que aumenta la amplitud de esfuerzos la vida por fatiga disminuye. Los esfuerzos alternativos provocaron que se propaguen las fisuras sobre la superficie de fractura.

Cuando se somete a torsión un eje circular, toda la sección transversal permanece plana [2]. Es decir, mientras las diferentes secciones a lo largo del eje rotan ángulos diferentes, cada sección transversal rota como una placa rígida [5]. De esta forma la distribución de las deformaciones cortantes varía linealmente con la distancia al centro del eje [4].

Los efectos de los parámetros de corte (velocidad de corte, avance y profundidad de corte) durante el maquinado están muy bien documentados y un gran número de estudios se han realizado para estudiar los efectos de la rugosidad producida por el maquinado en el rendimiento de piezas mecánicas sometidas a fatiga [15], [14]. La capa superficial se somete a deformación elasto-plástica, calentamiento (que resulta en cambios estructurales), endurecimiento por deformación y estreses residuales [16].

De la Tabla 1 obtenemos los valores de potencia y torque del vehículo y de la Figura 13 las dimensiones de la sección fallada.

TABLA 1 Datos técnicos tomados de Manual del usuario Toyota

\begin{tabular}{|c|c|}
\hline Marca & Toyota \\
\hline Modelo & Landcruiser Estate \\
\hline Año de Producción & 1976 \\
\hline Refrigerante & Agua \\
\hline Tipo de motor & OHV \\
\hline Número de cilindros & 6 en línea \\
\hline
\end{tabular}

\begin{tabular}{|c|c|}
\hline Válvulas por Cilindro & 2 \\
\hline Desplazamiento del motor & $4230 \mathrm{cc}$ \\
\hline Diámetro cilindro & $94 \mathrm{~mm}$ \\
\hline Carrera del pistón & $102 \mathrm{~mm}$ \\
\hline Relación Diámetro / Desplaza- \\
miento & 0.92 \\
\hline Capacidad Unitaria & $705 \mathrm{cc} / \mathrm{cilindro}$ \\
\hline Relación de Compresión & $7.8: 1$ \\
\hline Sistema de combustible: & Tipo 1 Carburador \\
\hline Torque Máximo a Potencia \\
Máxima
\end{tabular}

Fuente:http://www.carfolio.com/specifications/models/ car/?car $=53207$

FIG 13. DIMENSIONES DE LA SECCIÓN FALLADA

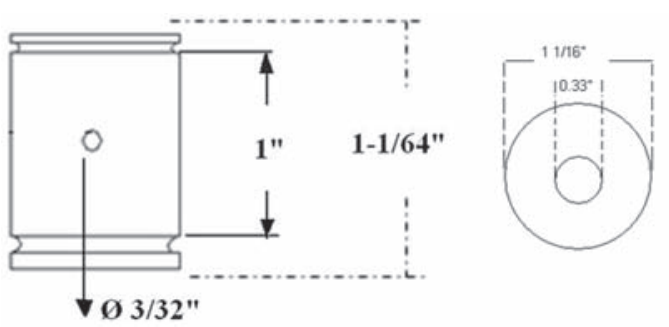

Fuente: Los autores

Se sabe a partir de la experiencia y de la literatura técnica pertinente, que los aceros más apropiados para la construcción de elementos de máquinas como este eje son los aceros al carbono de la serie AISI 10XX y los aceros aleados de las series AISI 41XX y AISI 43XX con un contenido de carbono no menor a 0,4 \% [3]. Los aceros más comúnmente utilizado son el AISI 1045, AISI 4140 y AISI 4340 que son aceros fácilmente disponibles en Colombia.

Mediante espectrometría de fluorescencia de Rayos X - XRF se determinó la composición química de los elementos aleantes, con el fin de caracterizar el tipo de acero presente. De acuerdo a este análisis químico, el acero con el cual se fabricó este eje se clasificó como un acero AISI 4140. Se 
toma como parámetro concluyente la baja cantidad de níquel presente que hizo descartar la serie de aceros AISI 43XX.s. Acorde con esto y usando para el acero AISI 4140 tenemos [13] se ve en la ecuación (1)

$$
\begin{gathered}
K_{\mathrm{IC}}=90 \mathrm{Mpa} \sqrt{(\mathrm{m})} \\
\sigma_{\mathrm{ic}}=1400 \mathrm{MPa} \\
K_{\mathrm{IC}}=\sigma_{\mathrm{I}} \sqrt{(\pi)(a)} \\
a_{c}=\left((90 / 1400)^{2}\right) / \pi
\end{gathered}
$$

Es decir, que el tamaño de la grieta máxima permisible para este esfuerzo es de 1,31546 mm

Ahora según las características geométricas del eje, sabemos que:

$$
\begin{aligned}
& \mathrm{K}=3 \sigma \Rightarrow \sigma=(K / 3)=\left(K_{\mathrm{IC}}\right) / 3 ; \\
& \sigma=(90 / 3)=30 \mathrm{MPa}
\end{aligned}
$$

De tal forma que el esfuerzo teórico máximo que soporta el eje con un factor de concentración de esfuerzos de.90 MPa $\sqrt{(\mathrm{m})}$

Ahora según la teoría de grietas de Griffith el factor de intensidad de esfuerzo $(\mathrm{K})$ para este caso está dado por la ecuación (3):

Con lo cual se demuestra la sobrecarga debido a

$$
\begin{aligned}
& K=1,12(3 \sigma) \quad(\pi)(L) \\
& \sigma_{\text {intinauta }}=90 /(1,12 \times 3 \sqrt{(\pi)(L)}) \\
& \sigma_{\text {mitimani }}=26,7857 / \sqrt{(0,0006 \pi)} \\
& \sigma_{\text {mitranio }}=616,955 \mathrm{MPa}
\end{aligned}
$$

la propagación de una grieta interna proveniente del conducto central, esto debido a las condiciones de carga ya mencionadas que producen esfuerzos alternantes producto de la operación normal del árbol de levas.

Según los datos técnicos obtenidos del vehículo, se puede calcular el esfuerzo cortante máximo (ecuación 4) que soportará el eje bajo las condiciones de torque máximo, es decir, $285 \mathrm{~N}-\mathrm{m}$ a $1800 \mathrm{rpm}$ para un eje hueco circular.

$$
\begin{aligned}
& \tau_{\max }=(\text { Torque }) \times(\mathrm{R} / \mathrm{J}) \\
& \tau_{\max }=285 \mathrm{~N} . \mathrm{m} \times(13,49 \mathrm{~mm}) /\left(51589,54 \mathrm{~mm}^{4}\right) \\
& \tau_{\max }=74,5 \mathrm{MPa}
\end{aligned}
$$

En las Figuras 5 y 6 se observan dos gargantas en los extremos del muñón. La Figura 15 nos da las dimensiones para este chavetero.

FIG 15. DIMENSIONES DE LA GARGANTA EN LOS EXTREMOS DEL MUÑÓN.

$r$

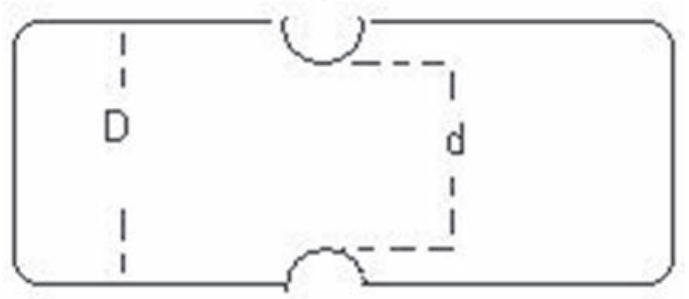

Fuente: Los autores

En donde:

$$
\begin{aligned}
& D=2,6987 \mathrm{~cm} ; d=2,560 \mathrm{~cm} ; \mathrm{r}=0,060 \mathrm{~cm} \\
& \mathrm{r} / \mathrm{d}=0,0234375 ; D / d=1,0541
\end{aligned}
$$

De acuerdo [8] el factor de concentración de esfuerzo Kt para este caso es de 2,2. Se asumió este valor como el valor total de $\mathrm{K}$.

Donde $(1,12) 2$ es el factor de corrección de superficie de defecto. Se despeja el esfuerzo y tenemos en la ecuación 6:

$$
\begin{aligned}
& \sigma=2,2 /(1,12)^{2} \times(2 / \pi) \sqrt{a \pi} \\
& \sigma=2,2 / 0,798575 \times \sqrt{0,00060 \times \pi} \\
& \sigma=63,455 \mathrm{MPa}
\end{aligned}
$$

\section{CONCLUSIONES}

El eje presenta fractura frágil debido a una sobre carga producida por un torque excesivo. La presencia de grietas, emanadas del conducto central, crearon las condiciones propicias para la falla.

La falla se localizó directamente sobre la zona en la cual se encuentra un cambio de sección, el cual a pesar de no tener un radio brusco ni agudo, si presenta entalladuras producidas por maquinado. La profundidad de este maquinado es excesivo y este crea una zona de altos concentradores de esfuerzos, ya que en una sección de solo 1/64" se alojan hasta 12 líneas de maquinado.

Se descarta la presencia de falla por fatiga ya que no hay presencia de marcas de playa ni secciones lisas. Igualmente se descarta la fatiga térmica como causante de falla ya que no hay indicios de secciones que presenten fragilidad en azul. 
No se observa presencia macroscópica de deformación plástica.

Las características de este acero y la temperatura de trabajo $\left(200-600{ }^{\circ} \mathrm{C}\right)$ descartan la falla por choque térmico. Al igual que en fatiga térmica no hay presencia de fragilidad en azul ni de contracciones o pandeos producto de un cambio de temperatura súbito.

Se encontró un alto valor del factor de concentración de esfuerzos localizado en los cambios de sección para este diseño. Además la presencia de entallas en esta misma sección facilitaron la rotura por esta parte de la pieza.

Para un estudio más detallado se sugiere analizar los cojinetes de la culata del motor con el fin de encontrar indicios de posibles cargas de flexión o anomalías superficiales que puedan aportar datos a esta investigación.

Es recomendable realizar un estudio de elementos finitos para corroborar la causa de falla propuesta acorde al criterio de Griffith.

\section{REFERENCIAS}

[1] Changli, W, Chengie, Z. Deping, W. "Analysis of an unusual crankshaft failure". Engineering Failure Analysis. Vol. 12, 2005

[2] Beer, F; Jhonston R., "Mecánica de Materiales".4. Ed.Mac-Graw Hill , México, 2001

[3] Dieter , G “Metalurgia Mecánica”.Ed. Aguilar , Barcelona,1967

[4] Mott, R. “Diseño de elementos de máquinas". Ed Prentice Hall \& IBD, 1995

[5] Hernández, H.; Espejo, E. “Mecánica de fractura y análisis de falla”. Ed Universidad Nacional de Colombia, Unibiblos. Bogotá, 2003

[6] Hertzberg, R. W. "Deformation and Mechanics of Engineering Materials". Editorial John Wiley \& Sons, Inc. 4 Edición, 1995

[7] Valdés, J. A.; Coronado, J. J.; García, J. I., “Comparación Y Estudio de la Fractura del Cigüeñal de un Motor de Cuatro Cilindros en Línea”. Scientia Et Technica Año XII, No 31, Agosto 2006

[8] ASM, Metals Handbook, 9a edición. Cleveland 1999.

[9] Mayz, E. Conocimientos Básicos del Automóvil. Octubre 2008: Disponible en: h ttp://www.automotriz.net/ tecnica/conocimientos-basicos-04.html
[10] Planells, R; Amengual, Á.; Larruy, A. "Motores de combustión interna: Fundamentos";. Ed. UPC, Barcelona, 1994

[11] Meyers, M. A; Chawla K., "Mechanical Behavior of Materials”, 1 ed, Prentice Hall, New Jersey, 1999

[12] Anderson, T. "Fracture Mechanics", 2 ed, CRC press, Boca Raton, 1995

[13] Compañía General de Aceros, Catálogo de productos 2008, Bogota

[14] Yang, W. and Tarng, Y., “Design Optimization of Cutting Parameters for Turning Operations based on Taguchi Method", J. Mater. Process. Technol., Vol. 84, 1998

[15] Trent, E. M. and Wright, P. K., "Metal Cutting”, Ed. Butteworths- Heinemann Ltd., 2000, London, England

[16] Lopes K., Sales W., Palma E., Influence of Machining Parameters on Fatigue Endurance Limit of AISI 4140 Steel. J. of the Braz. Soc. of Mech. Sci. \& Eng., No. 1, 2008 